\title{
Observation of acoustic turbulence in a system of nonlinear second sound waves in superfluid ${ }^{4} \mathrm{He}$
}

\author{
A.N. Ganshin ${ }^{1}$, V.B. Efimov ${ }^{1,2}$, G.V. Kolmakov ${ }^{1,2}$, \\ P.V.E. McClintock ${ }^{1}$, and L.P. Mezhov-Deglin ${ }^{2}$ \\ ${ }^{1}$ Department of Physics, Lancaster University, Lancaster, LA1 4YB, UK \\ ${ }^{2}$ Institute of Solid State Physics RAS, Chernogolovka, \\ Moscow region, 142432, Russia
}

A paper prepared for the special issue of Low Temperature Physics "Helium: 100 years".

Running title: Acoustic turbulence in superfluid ${ }^{4} \mathrm{He}$ PACS: 67.40.Pm, 47.27.Cn, 05.20.Dd 


\begin{abstract}
We discuss the results of recent studies of acoustic turbulence in a system of nonlinear second sound waves in a high-quality resonator filled with superfluid ${ }^{4} \mathrm{He}$. It was found that, when the driving amplitude was sufficiently increased, a steady-state direct wave cascade is formed involving a flux of energy towards high frequencies. The wave amplitude distribution follows a power law over a wide range of frequencies. Development of a decay instability at high driving amplitudes results in the formation of subharmonics of the driving frequency, and to a backflow of energy towards the low-frequency spectral domain, in addition to the direct cascade.
\end{abstract}

Keywords: Superfluid helium, second sound, acoustic turbulence, Kolmogorov spectrum 


\section{Introduction}

First, we are much indebted to the Editorial Board of the Low Temperature Physics journal for their invitation to report the results of our recent studies in this special issue to mark the $100^{\text {th }}$ anniversary of the liquefaction of helium.

In what follows, we review our experimental investigations of the acoustic turbulence created in a system of nonlinear second sound standing waves in a high-quality resonator filled with He II, the superfluid phase of ${ }^{4} \mathrm{He}$.

It is well-known that He II supports two quite separate sound propagation modes: first sound (conventional pressure/density waves) and second sound (temperature/entropy waves) $[1,2]$. Second sound waves of infinitely small amplitude running in bulk superfluid He II are characterized by a linear dispersion relation between frequency $\omega$ and wave vector $k[2]$

$$
\omega_{k}=u_{20} k
$$

which is also typical of acoustic waves in condensed matter and in gases. Here $u_{20}$ is the second sound velocity, which depends on the temperature of the helium sample. At temperatures close to that of the superfluid-to-normal transition at $T_{\lambda}=2.177 \mathrm{~K}$, the velocity $u_{20}$ tends to zero. At temperatures down to $T \sim 1 \mathrm{~K}$, the second sound velocity is $u_{20} \leq 20 \mathrm{~m} / \mathrm{s}$, which is much smaller than the velocity of conventional sound in condensed media, $u_{10} \geq 3 \times 10^{2} \mathrm{~m} / \mathrm{s}$. We emphasize that in contrast to ordinary (classical) media, where a temperature wave is damped over a distance of the order of its wavelength [1], the second sound wave mode has very small dissipation within the experimentally convenient temperature range $T \sim 1.5-2.1 \mathrm{~K}$ : typically, a second sound pulse of duration $\tau \sim 1 \mu$ s (i.e. of wavelength $\lambda \sim 2 \times 10^{-3}$ $\mathrm{cm})$ can propagate through the superfluid over a distance of metres before being damped by viscous losses.

Second sound is characterized by rather strong nonlinear properties $[2,3,4]$. For example, a traveling second sound pulse of amplitude $\delta T \sim 1 \mathrm{mK}$ (i.e. with relative amplitude $\delta T / T \sim 10^{-3}$ ) transforms into a shock wave over a distance $L<1 \mathrm{~cm}$ 
from the source $[5,6,7]$. To a first approximation, the velocity of a second sound wave of finite amplitude depends on the wave amplitude $\delta T$ as

$$
u_{2}=u_{20}(1+\alpha \delta T)
$$

Here

$$
\alpha=\frac{\partial}{\partial T} \ln \left(u_{20}^{3} \frac{C}{T}\right)
$$

is the nonlinearity coefficient of second sound and $C$ is the specific heat per unit mass at constant pressure. Under the saturated vapor pressure, in the region of roton second sound, $T>0.9 \mathrm{~K}$, the nonlinearity coefficient is positive $(\alpha>0)$ for temperatures $T<T_{\alpha}=1.88 \mathrm{~K}$ (like the nonlinearity coefficient of conventional sound waves in ordinary media); but it is negative in the range $T_{\alpha}<T<T_{\lambda}$ (and many times larger than the nonlinearity coefficient of first sound) [4]. At $T=T_{\alpha}$ the nonlinearity coefficient passes through zero.

In the studies reported below, we exploit these special properties of second sound in He II for an investigation of turbulence in a system of nonlinear acoustic waves (acoustic turbulence). This is a state in which a large number of acoustic wave modes are excited and interacting strongly with each other. It is is characterized by a directed energy flux through frequency scales $[8,9,10,11]$.

Acoustic turbulence has been at the focus of numerous investigations during the last few decades because of its importance for basic nonlinear physics and in view of numerous applications in engineering and fundamental science [8]. Well known examples of acoustic turbulence include the turbulence of sound waves in oceanic waveguides [12], magnetic turbulence in interstellar gases [13], and shock waves in the solar wind and their coupling with the Earth's magnetosphere [14].

Second sound is ideal for modelling the dynamics of nonlinear waves because of the way in which its nonlinearity coefficient, which determines the strength of the wave interactions, can be tuned over a wide range simply by changing the bath temperature. It allows one to study the dynamics of both nearly linear and strongly nonlinear waves, with both positive (like conventional sound) and negative non- 
linearity, while using exactly the same experimental techniques. Such possibilities are unavailable in conventional experiments. The fact that the velocity of second sound $u_{20}$ is relatively small permits one to increase time resolution of the measurements. Note that the acoustic turbulent state is radically different from quantum turbulence (QT) [15], which is also formed in He II, because the density of quantized vortices is close to zero. Furthermore, the motions of both the normal and superfluid components can be considered as being to a first approximation potential.

Based on measurements of nonlinear second sound waves in a high-quality resonator, we observed formation of a steady-state wave-energy cascade in He II involving a flux of energy through the spectral range towards high frequencies. Initial results of the studies were published in Ref. [11]. Since then, we have found that, under some circumstances, wave energy in the acoustic system can also flow in the reverse direction. Below we discuss these observations in more detail.

\section{Experimental techniques}

The experimental arrangements were similar to those used in our earlier studies of nonlinear second sound waves $[16,17]$. The cryoacoustical resonator was made of a cylindrical quartz tube of nominal length $L=7 \mathrm{~cm}$ and internal diameter $D=1.5$ $\mathrm{cm}$, filled with superfluid helium. The low-inertial film heater and bolometer were deposited on the surfaces of flat glass plates capping the ends of the tube. The heater was driven by a harmonic voltage generator in the frequency range $0.1-100 \mathrm{kHz}$. The frequency of the second sound (twice the frequency of the voltage generator) was set close to the frequency of a longitudinal resonance in the resonator. The amplitude of the standing wave $\delta T$ could be changed from $0.05 \mathrm{mK}$ up to a few $\mathrm{mK}$ by adjustment of the power to the heater. The measured heat flux density $W$ into the liquid is subject to systematic uncertainties of up to $\pm 10 \%$ associated with estimation of the resonator cross-section and the resistances of the leads, and possible small inhomogeneities in the heater film thickness. The $Q$-factor of the resonator 
determined from the widths of longitudinal resonances at small heat fluxes $W \leq 4$ $\mathrm{mW} / \mathrm{cm}^{2}$ (nearly linear regime) was $Q \sim 3000$ for resonance numbers $20<p<100$ and decreased to about 500 at frequencies below $500 \mathrm{~Hz}$. A typical resonance curve is shown in Fig. 1 (the 32nd resonance).

Use of a high- $Q$ resonator enables us to create nonlinear second sound standing waves of high amplitude ( $\delta T \sim 1 \mathrm{mK})$ accompanied by only small heat input at the source $W<55 \mathrm{~mW} / \mathrm{cm}^{2}$, thus avoiding possible complications [18] due to vortex creation in the bulk He II and nonlinear phenomena at the heater/superfluid interface. The second sound waveform registered by the bolometer was Fourieranalyzed and its power spectrum was computed.

\section{Results and discussion}

Fig. 2 shows the evolution of the second sound wave spectrum with increasing AC heat flux density $W$ from the heater, measured at temperature close to $2.08 \mathrm{~K}$ when driving at the frequency of the 31st resonance. For small $W \leq 4 \mathrm{~mW} / \mathrm{cm}^{2}$ we observed a nearly linear regime of wave generation, where a small number of harmonics of the driving frequency were excited due to nonlinearity (see Fig. 2(a)), and the shape of the recorded signal was close to sinusoidal. An increase of the excitation above $12 \mathrm{~mW} / \mathrm{cm}^{2}$ led to visible deformation of the signal shape and to the generation of a large number of harmonics in the second sound wave spectrum as shown in Fig. 2(b).

It is evident from Fig. 2 that the main spectral peak (marked by the arrow) lies at the driving frequency $\omega_{d}$, and that high-frequency peaks appear at its harmonics $\omega_{n}=n \times \omega_{d}$ with $n=2,3, \ldots$ It can be seen in Fig. 2(b) that a cascade of waves is formed over the frequency range up to $80 \mathrm{kHz}$, i.e. up to a frequency 25 times higher than the driving frequency. As also shown in Fig. 2(b), the dependence of peak height on frequency may be described by a power-law-like function $\delta T_{\omega}=$ const $\times \omega^{-s}$ for frequencies lower than some cut-off frequency $\omega_{b}$ that increases with increasing $W$. 
Note there are systematic uncertainties of about $\pm 10 \%$ in the values of $s$ and $\omega_{b}$ extracted from plots of this kind, depending on the range of $\omega$ through which the straight line is drawn. For sufficiently high AC heat flux densities $W>12 \mathrm{~mW} / \mathrm{cm}^{2}$ (i.e. for the developed cascade), the scaling index tends towards $s \approx 1.5$.

Formation of the spectra observed in the experiments is evidently attributable to the cascade transfer of wave energy through the frequency scales due to nonlinearity, thus establishing an energy flux in $K$ space directed from the driving frequency towards the high-frequency domain. In accordance with basic ideas formulated in Refs. $[8,9,10]$ we may infer that, at relatively high driving amplitudes, we are observing acoustic turbulence formed in the system of second sound waves within the inertial (nondissipative) range of frequencies. Formation of the observed direct cascade is similar to creation of the Kolmogorov distribution of fluid velocity over frequency in the bulk of a classical fluid [19].

We observed also that, when the AC heat flux density was raised above some critical value at even resonance numbers $p>30$, a spectral peak appeared at the frequency equal to half the driving frequency (i.e. formed on the left of the fundamental peak) and at its harmonics. Fig. 3 shows the evolution of the wave spectrum with increasing $\mathrm{AC}$ heat flux density when driving on the $32 \mathrm{nd}$ resonance. It is evident that, at the relatively small heat flux density $W=4 \mathrm{~mW} / \mathrm{cm}^{2}$, the wave spectrum shown in Fig. 3(a) is quite similar to that observed under similar conditions when driving at the 31st resonance (i.e. at the nearest odd numbered resonance), see Fig. 2(a). Formation of the low-frequency harmonic (subharmonic) at $\omega=\omega_{d} / 2$ at $W=16 \mathrm{~mW} / \mathrm{cm}^{2}$ is clearly seen in Fig. 3(b). It was found that the threshold value for generation of subharmonics obtained in measurements with $30<p<95$ was about $12 \mathrm{~mW} / \mathrm{cm}^{2}$.

The formation of subharmonics may be attributed to development of a decay instability of the periodic wave. In accordance with general theory [8, 20, 21], the instability is controlled mainly by nonlinear decay of the wave into two waves of lower frequency, and by the opposite process of the confluence of two waves to form 
one wave. The energy (or frequency) conservation law for this 3-wave process is

$$
\omega_{1}=\omega_{2}+\omega_{3},
$$

where $\omega_{i}=u_{20} k_{i}$ is the frequency of a linear wave of wave vector $k_{i}$. The case shown in Fig. 3(b) evidently corresponds to generation of subharmonics with $\omega_{2}=\omega_{3}=$ $\omega_{d} / 2$.

We also observed the high-frequency cutoff of the wave spectrum due to viscosity. As shown in Fig. 2(a), it manifests itself as an abrupt decrease in the amplitudes of the harmonics at $W<12 \mathrm{~mW} / \mathrm{cm}^{2}$, and as a change in slope of the spectrum at higher $W$ (Fig. 2(b)) when plotted on double-log scales, which occurs at some characteristic frequency $\omega_{b}$. At $\omega \sim \omega_{b}$ the nonlinear mechanism for nearly nondissipative transfer of the wave energy changes to viscous damping of the waves (cf. observations of the high-frequency edge of the inertial range of frequencies of capillary turbulence on the surface of liquid hydrogen [22]). It causes a faster reduction of sound amplitudes at frequencies $\omega>\omega_{b}$, as observed.

The dependence of the boundary frequency $\omega_{b} / 2 \pi$ on the standing wave amplitude $\delta T$ is shown in Fig. 4. It is seen that the inertial range is extended towards higher frequencies when the driving force is increased. When driving at resonant frequencies with odd resonance numbers $p$, with sufficiently large driving amplitudes, the boundary frequency depends linearly on wave amplitude

$$
\omega_{b} / 2 \pi=\operatorname{const}\left(T, \omega_{d}\right) \times \delta T,
$$

(the filled symbols in Fig. 4) in agreement with our numerical calculations [11].

When driving at even $p$ (open symbols in Fig. 4) the boundary frequency is noticeably lower than that measured for the nearest odd resonance number, with $W>12 \mathrm{~mW} / \mathrm{cm}^{2}$. This reduction may be connected with a change in the mechanism of energy relaxation in the wave system caused by the generation of subharmonics with frequencies lower than $\omega_{d}$.

One can see from Fig. 4 that the energy balance in the wave system is highly nonlocal in $K$ space: energy is pumped into the system in the low-frequency (long- 
wave) domain and it flows to the high frequency (short-wave) domain where it is absorbed by dissipative mechanisms.

It was found that, when driving at sufficiently high AC heat flux densities $W>23$ $\mathrm{mW} / \mathrm{cm}^{2}$ and at resonance numbers $p>50$, multiple subharmonics were generated in the low-frequency spectral domain $\omega<\omega_{d}$, see Fig. 5. Here we present the initial results of our study of the decay instability in the acoustic system, a phenomenon that promises to be of huge interest for nonlinear sound wave dynamics. The results of the more detailed investigations now in progress will be published and discussed elsewhere in due course.

The formation of subharmonics in the wave spectrum is evidently attributable to further development of the decay instability, when not only a wave with frequency $\omega_{d} / 2$ is created due to nonlinearity, but a number of waves with frequencies obeying the conservation law (2) are generated. This regime is quite similar to the kinetic instability known for weak turbulent systems [21]. It is seen from Fig. 5 that, when the instability develops, the wave spectrum becomes almost continuous: all possible modes seem to be excited.

We may interpret the generation of waves of frequency lower than the driving frequency as the establishment of an energy backflow towards low frequencies. Inverse energy cascades are known in 2-dimensional incompressible liquids [23] and Bose gases [24] but, to our knowledge, this phenomena has not been observed earlier for nonlinear acoustic waves. Absorption of the wave energy at low frequencies is probably attributable to viscous drag of the normal fluid component on the resonator walls, given that bulk second sound damping is negligibly small in this frequency range: this would be consistent with the observed strong decrease of the resonator $Q$-factor below $2 \mathrm{kHz}$. We have also observed that formation of the inverse energy cascade is accompanied by a reduction of wave amplitude in the high frequency spectral domain, and by a contraction of the inertial range. This observation is consistent with our inference that, after onset of the instability, the energy flux is shared between the direct and inverse energy cascades. 


\section{Conclusions}

We have demonstrated that the system of second sound waves in a high- $Q$ resonator filled with He II can be used as an effective tool for the detailed modelling and investigation of acoustic turbulence. We observed a smooth crossover in the system of second sound waves from a nearly linear regime at low driving amplitudes to a nonlinear regime at moderate driving amplitudes, and, further, to developed turbulence at high driving amplitudes (a Kolmogorov-like cascade). In the high frequency domain a cutoff of the cascade is observed, caused by a change in the mechanism of energy transfer, from nonlinear wave transformation to viscous damping. When driving at moderate amplitudes at resonances of even resonance number, a decay instability develops in the system due to 3 -wave interactions. It results in the generation of a subharmonic of frequency equal to half the driving frequency. At relatively high driving amplitudes multiple subharmonics are generated in the wave spectrum, corresponding to the formation of an inverse energy cascade directed towards the low-frequency spectral domain.

\section{Acknowledgments}

The investigations were supported by the Russian Foundation for Basic Research, project Nos. 06-02-17253 and 07-02-00728, by the Presidium of the Russian Academy of Sciences in frames of the programs "Quantum Macrophysics" and "Mathematical Methods in Nonlinear Dynamics", and by the Engineering and Physical Sciences Research Council (UK). 


\section{References}

[1] L. D. Landau and E. M. Lifshits, Fluid Mechanics, 2nd ed., Pergamon Press, Oxford (1987).

[2] I. M. Khalatnikov, An Introduction to the Theory of Superfluidity, Benjamin, New York (1965).

[3] D. V. Osborne, Proc. Phys. Soc. (London) A 64, 114 (1951).

[4] A. J. Dessler and W. M. Fairbank, Phys. Rev. 104, 6 (1956).

[5] A. Yu. Iznankin and L. P. Mezhov-Deglin, Sov. Phys. JETP 57, 801 (1983).

[6] L. S. Goldner, G. Ahlers, and R. Mehrotra, Phys. Rev. B 43, 12861 (1991).

[7] G. V. Kolmakov, V. B. Efimov, A. N. Ganshin, P.V.E. McClintock, E. V. Lebedeva, and L. P. Mezhov-Deglin, Low Temp. Phys. 32, 981 (2006).

[8] V. E. Zakharov, G. Falkovich, and V. S. L'vov, Kolmogorov Spectra of Turbulence I, Springer, Berlin (1992).

[9] V. E. Zakharov and R. Z. Sagdeev, Doklady Acad. Nauk SSSR 192, 297 (1970).

[10] G. Falkovich and M. Meyer, Phys. Rev E 54, 4431 (1996).

[11] G. V. Kolmakov, V. B. Efimov, A. N. Ganshin, P. V. E. McClintock, and L. P. Mezhov-Deglin, Phys. Rev. Lett. 97, 155301 (2006).

[12] S. N. Gurbatov, V. V. Kurin, L. M. Kustov, and N. V. Pronchatov-Rubtsov, Acoust. Phys. 51, 152 (2005).

[13] G. S. Bisnovatyi-Kogan and S. A. Silich, Rev. Mod. Phys. 67, 661 (1995).

[14] M. Ryutova and T. Tarbell, Phys. Rev. Lett. 90, 191101(2003).

[15] W. F. Vinen, J. Low Temp. Phys. 145, 7 (2006). 
[16] I. Yu. Borisenko, V.B. Efimov, L.P. Mezhov-Deglin, Sov. J. Low Temp. Phys. 14, 619 (1988).

[17] V. B. Efimov, A. N. Ganshin, P. V. E. McClintock, G. V. Kolmakov and L. P. Mezhov-Deglin, J. Low Temp. Phys. 145, 155 (2006).

[18] H. Davydowitz, Yu. L'vov, and V. Steinberg, Physica D 84, 635 (1995).

[19] A. N. Kolmogorov, Doklady Akad. Nauk SSSR 30, 299 (1941).

[20] V. S. L'vov, Wave turbulence under parametric excitation. Application to magnetics, Springer, Berlin (1994).

[21] V. S. L'vov and V.B. Cherepanov, Sov. Phys. JETP 54746 (1981).

[22] G. V. Kolmakov, A. A. Levchenko, M. Yu. Brazhnikov, L. P. Mezhov-Deglin, A. N. Silchenko, and P. V. E. McClintock, Phys. Rev. Lett. 93 (7), 074501 (2004).

[23] R. H. Kraichnan, Phys. Fluids 10, 1417 (1967).

[24] S. Nazarenko and M. Onorato, J. Low Temp Phys. 146, 31 (2007). 


\section{Figure Captions}

Fig. 1. Second sound resonance curve measured in the He II filled resonator at a temperature close to $2.08 \mathrm{~K}$. The system was driven at a frequency close to its $32 \mathrm{nd}$ resonance with an AC heat flux density of $W=4 \mathrm{~mW} / \mathrm{cm}^{2}$.

Fig. 2. Evolution in the power spectrum of second sound standing waves as the AC heat flux density is increased from (a) $W=4 \mathrm{~mW} / \mathrm{cm}^{2}$ to (b) $25 \mathrm{~mW} / \mathrm{cm}^{2}$. The dashed line in (a) is a guide to the eye, whereas that in (b) corresponds to $\delta T \propto \omega^{-1.7}$. The arrows indicate the positions of the fundamental spectral peak formed at the driving frequency $\omega_{d}$ and of the high-frequency edge $\omega_{b}$ of the inertial frequency range. The system was driven at its 31st resonance, at a temperature close to $2.08 \mathrm{~K}$.

Fig. 3. Second sound wave spectra measured when driving on the frequency of the $32 \mathrm{nd}$ resonance with (a) $W=4 \mathrm{~mW} / \mathrm{cm}^{2}$ and (b) $W=16 \mathrm{~mW} / \mathrm{cm}^{2}$. The arrows indicate the fundamental peaks at the driving frequency $\omega=\omega_{d}$ and a subharmonic formed at $\omega=\omega_{d} / 2$. The temperature was close to $2.08 \mathrm{~K}$.

Fig. 4. Dependence of the viscous cutoff frequency $\omega_{b} / 2 \pi$ on the amplitude $\delta T$ of the standing wave for different temperatures $T$ and resonance numbers $p$. Dashed lines indicate the results of our numerical computations [11]; data points represent experimental measurements.

Fig. 5. A second sound wave spectrum measured when driving at the frequency of the 96th resonance with an AC heat flux density $W=25 \mathrm{~mW} / \mathrm{cm}^{2}$. The temperature was close to $2.08 \mathrm{~K}$. The arrow marks the fundamental peak at the driving frequency $\omega=\omega_{d}$. 


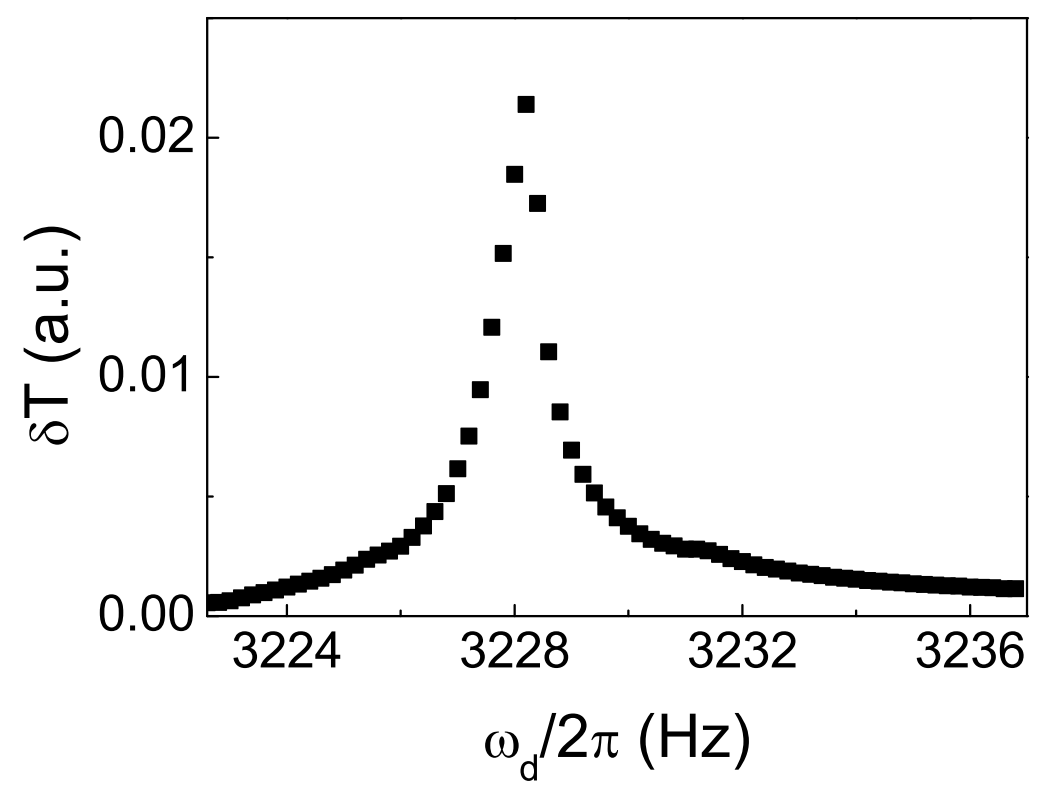

Figure 1: Ganshin et al, "Formation of acoustic turbulence..." 

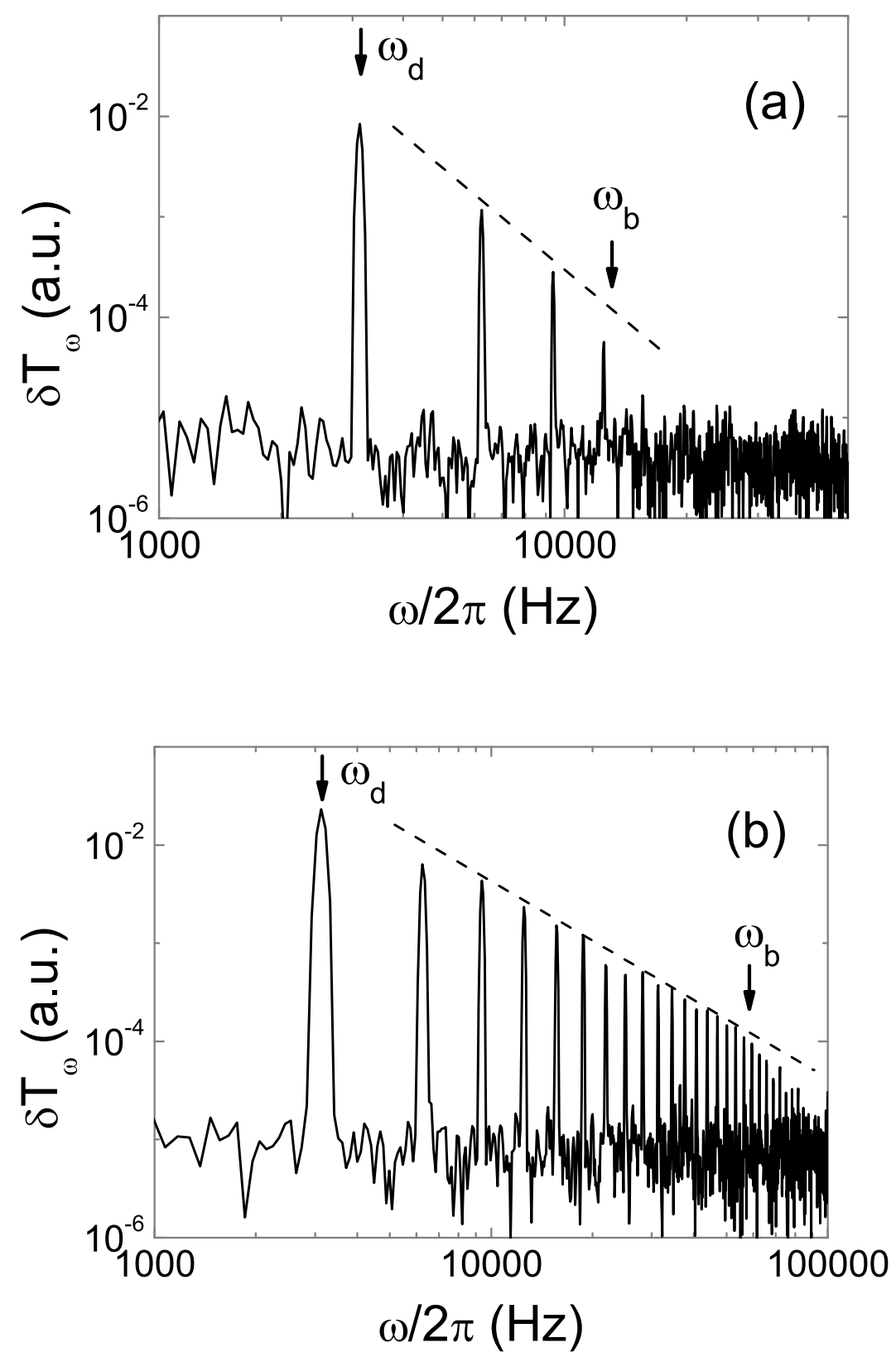

Figure 2: Ganshin et al, "Formation of acoustic turbulence..." 

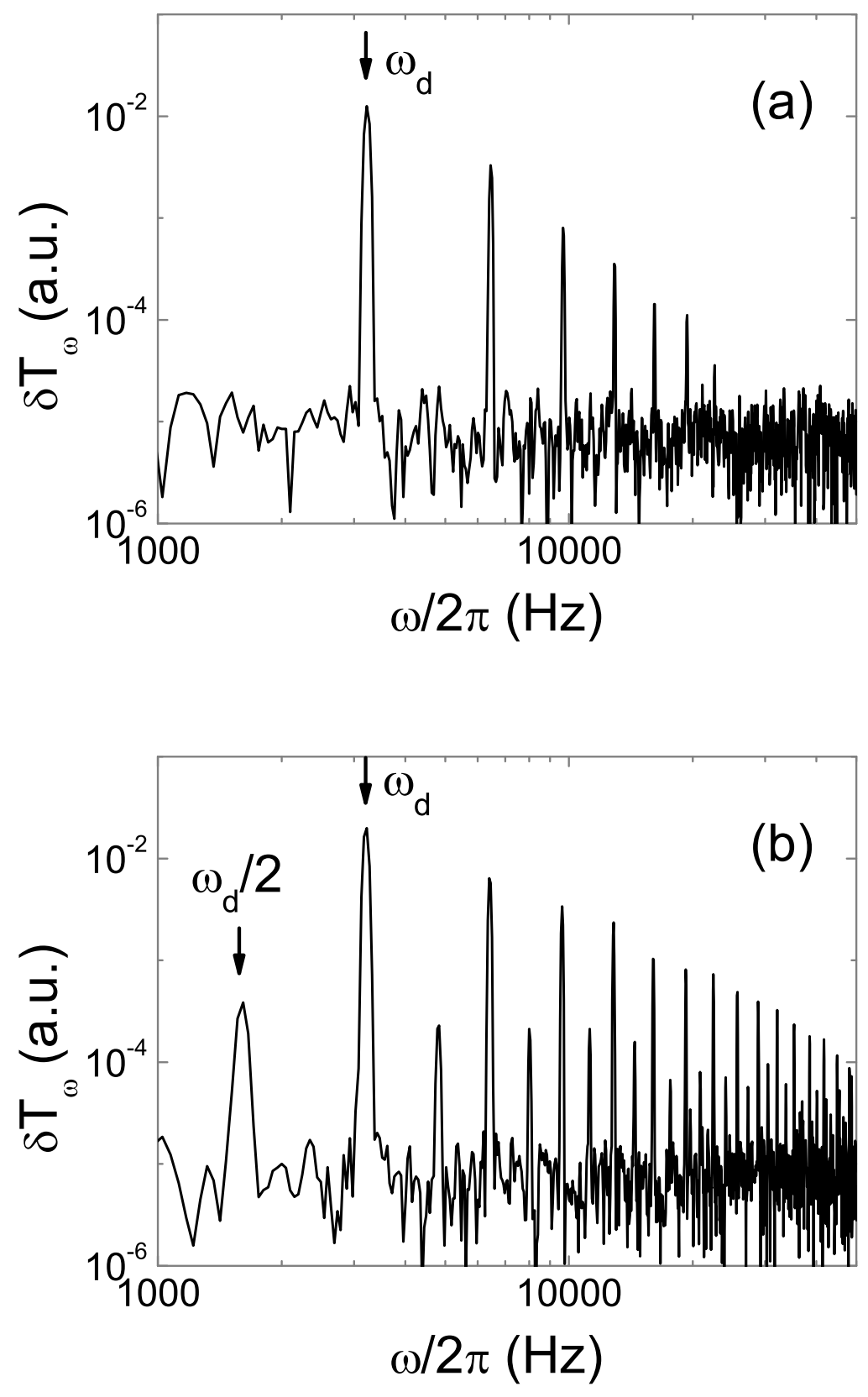

Figure 3: Ganshin et al, "Formation of acoustic turbulence..." 


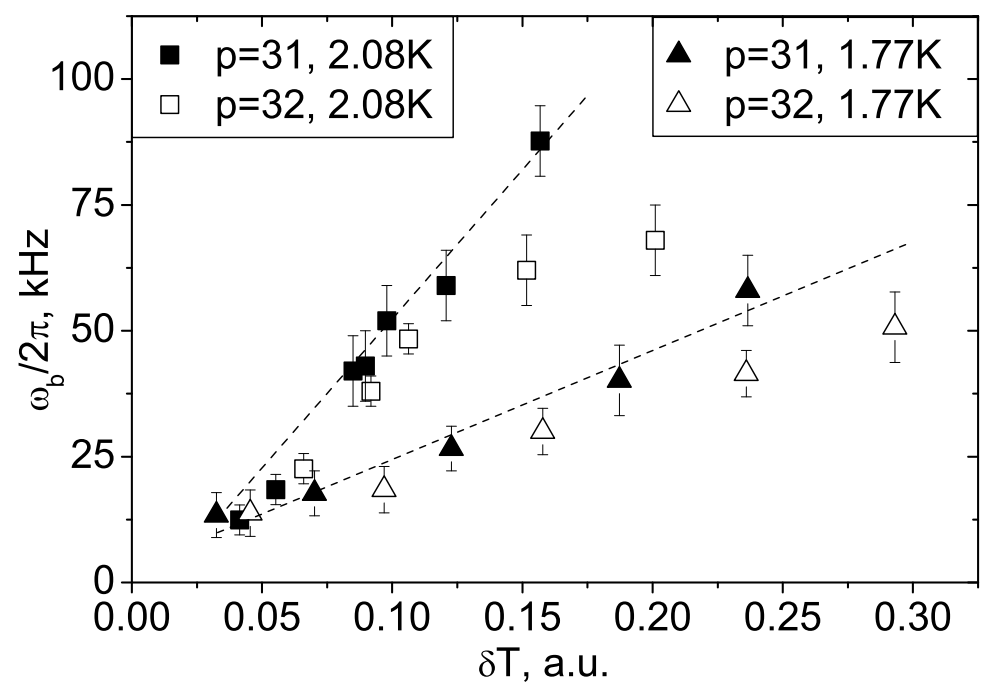

Figure 4: Ganshin et al, "Formation of acoustic turbulence..." 


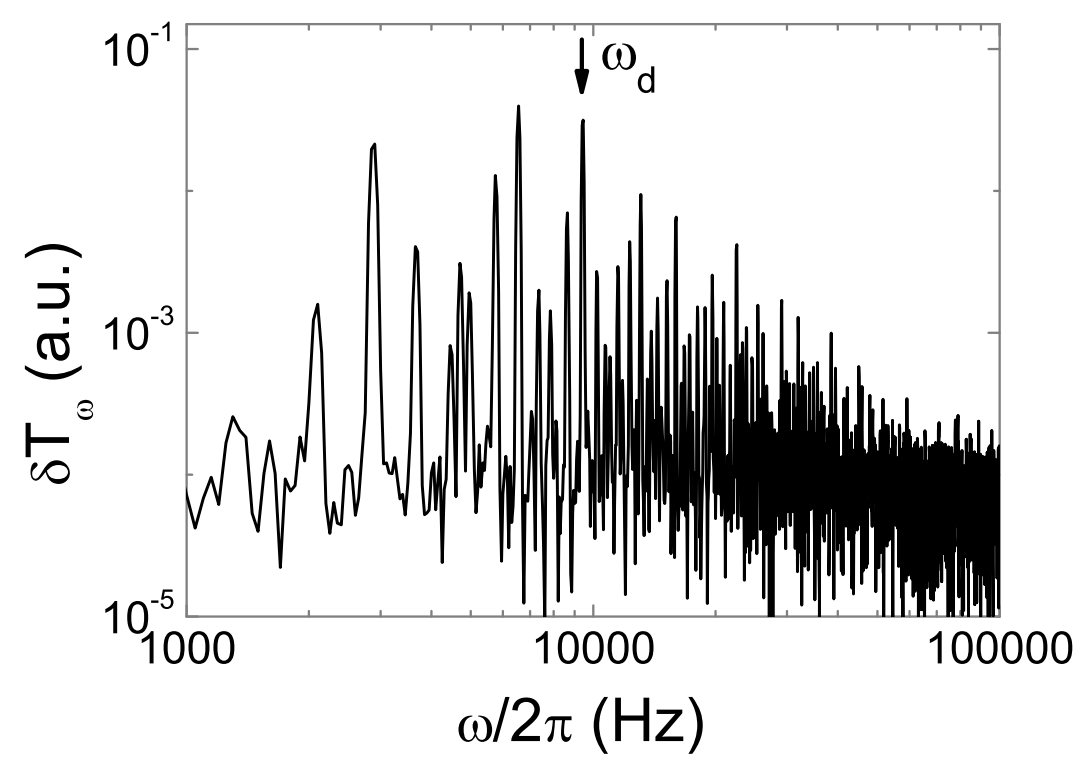

Figure 5: Ganshin et al, "Formation of acoustic turbulence..." 